\title{
Probing Properties of Nanomaterials with Advanced Electron Energy-Loss Spectroscopy
}

Peter Crozier ${ }^{1}$, Joshua Vincent ${ }^{2}$, Kartik Venkatraman ${ }^{3}$, Yifan Wang ${ }^{4}$ and Shize Yang ${ }^{2}$

${ }^{1}$ Arizona State University, Tempe, Arizona, United States, ${ }^{2}$ Arizona State University, United States,

${ }^{3}$ Center for Nanophase Materials Sciences, Oak Ridge National Laboratory, Oak Ridge, Tennessee 37831, USA, United States, ${ }^{4}$ School for the Engineering of Matter, Transport and Energy, Arizona State University, United States

Electron energy-loss spectroscopy (EELS) is a powerful tool for characterizing the composition and properties of materials at the nanometer level. Developments over the last 10 years in the areas of monochromators, spectrometers, and electron detectors now make it possible to probe excitations in solids from the meV to $\mathrm{keV}$ range, with atomic resolution possible in many cases. The types of excitations that can be probed with EELS includes phonons, polaritons, plasmons, excitons, photonic modes, interband and bandgap transitions, as well as inner-shell transitions to states above the Fermi level. This potentially provides a huge amount of local information about vibrational, electronic, optical, and magnetic properties of the materials. Many of these signals can also be acquired from samples under in situ or operando conditions, providing critical links between dynamic changes in materials properties and functionalities [1]. Current detector technology can reach readout rates in excess of 3,000 spectra per second, enabling both high spatial and temporal resolution to be achieved. Emerging data science techniques such as machine learning will play a critical role in extracting information from very large spectral datasets. At Arizona State University, our advanced EELS capability is primarily focused on two machines. The Nion UltaSTEM provides an energy resolution of about $6 \mathrm{meV}$, enabling phononic, photonic, and bandgap states to be probed with atomic resolution. We recently installed a Gatan Image Filter with a direct electron K3 camera on our FEI Titan environmental transmission electron microscope, dramatically improving our ability to perform EELS under in situ conditions. This presentation will highlight recent applications of these two machines to a variety of problems in materials characterization.

Vibrational spectroscopy is now routinely possible on the electron microscope with newly developed monochromators [2-4]. Atomic resolution has been achieved with a number of different approaches, although the underlying physics required for quantification must still be addressed $[2,5,6]$. The ability to generate high spatial resolution vibrational information that has features in common with both IR and neutron spectroscopy will open up completely new avenues for materials research. Figure 1 shows a spectrum from hexagonal $\mathrm{BN}$ which shows long wavelength dipole active modes (similar to those observed in IR) and short wavelength modes associated with the Brillouin zone boundary (BZB) modes similar to those observed in neutron scattering [7]. The high energy resolution and narrow zero-loss peak tail also increases the sensitivity for probing features that appear in the band gap of semiconductors and insulators, such as bandgap states, excitons, and photonic modes [8]. Many of these excitations play a key role in solar energy conversion processes and EELS not only allows such modes to be detected but can also sense coupling between different modes, allowing energy transport pathways to be explored. We have recently been using this approach to investigate the coupling between photonic modes and plasmons for photocatalytic applications.

In situ methods provide a dynamic view of how materials change and respond to external stimuli and understanding these dynamics is key to functionality. Time-resolved in situ EELS can provide a method 
to monitor dynamic materials properties. STEM EELS analysis provides a powerful approach to determine composition at the nanometer and atomic level, and the rapid readout rate that can be accomplished with the new detector enables chemical change to be tracked during in situ experiments. Energy filtered imaging (EFTEM) can also provide a complimentary approach to track chemical changes in samples under in situ conditions. Although it is more difficult to quantify than STEM EELS and the spatial resolution is typically a nanometer, EFTEM provides an alternative dark-field contrast mechanism that is sensitive to features in the low-loss as well as core-loss spectrum. One advantage of energy filtered imaging is that it allows a very large area of the sample to be observed rapidly with nanometer resolution. Figure 2 shows two energy filtered images acquired with the low-loss part of the spectrum from a cluster of $\mathrm{CeO}_{2}$ nanocubes. Figure $2 \mathrm{a}$ was recorded from the $35 \mathrm{eV}$ around the $\mathrm{CeO}_{2}$ bulk plasmon peak maximum whereas Figure $2 \mathrm{~b}$ was acquired from $25 \mathrm{eV}$, which is more sensitive to surface loss mode. The contrast in such images arise from structurally sensitive diffraction and chemically sensitive inelastic scattering [9]. Manipulating pairs of such images can suppress or enhance these different forms of contrast as shown in figure $2 \mathrm{c}$ where the (100) $\mathrm{CeO}_{2}$ surface is enhanced. For in situ measurements, it is often difficult to predict where a reaction or phase change may initiate. Being able to record chemically sensitive images with 3400 x 3400 size or larger in reasonable time can provide a spatio-temporal picture of changes taking place at time resolution not currently possible with STEM. We will show in situ examples where rapid, inelastic imaging of large areas provide information about materials changes that cannot be easily obtained with other approaches.

a

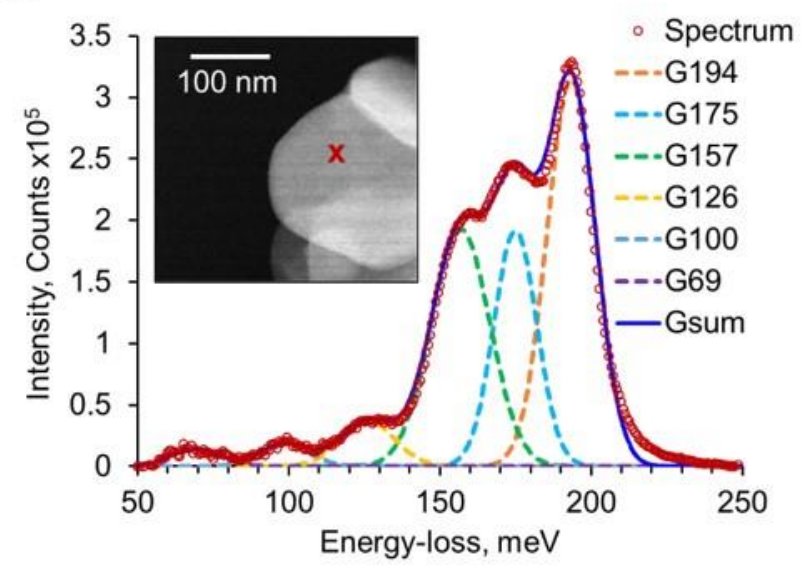

b

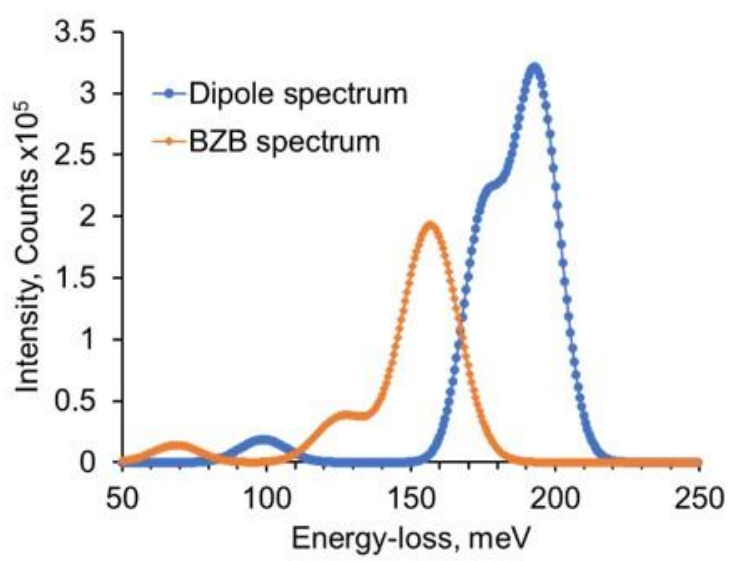

Figure 1. Figure 1. (a) Background subtracted vibrational spectrum with 33 mrad convergence and 10 mrad collection semi-angles. Gaussian fitting was also performed as shown. (Inset) ADF image of the nanoparticle showing the probe position. (b) Dipole and BZB contribution to the spectrum. 

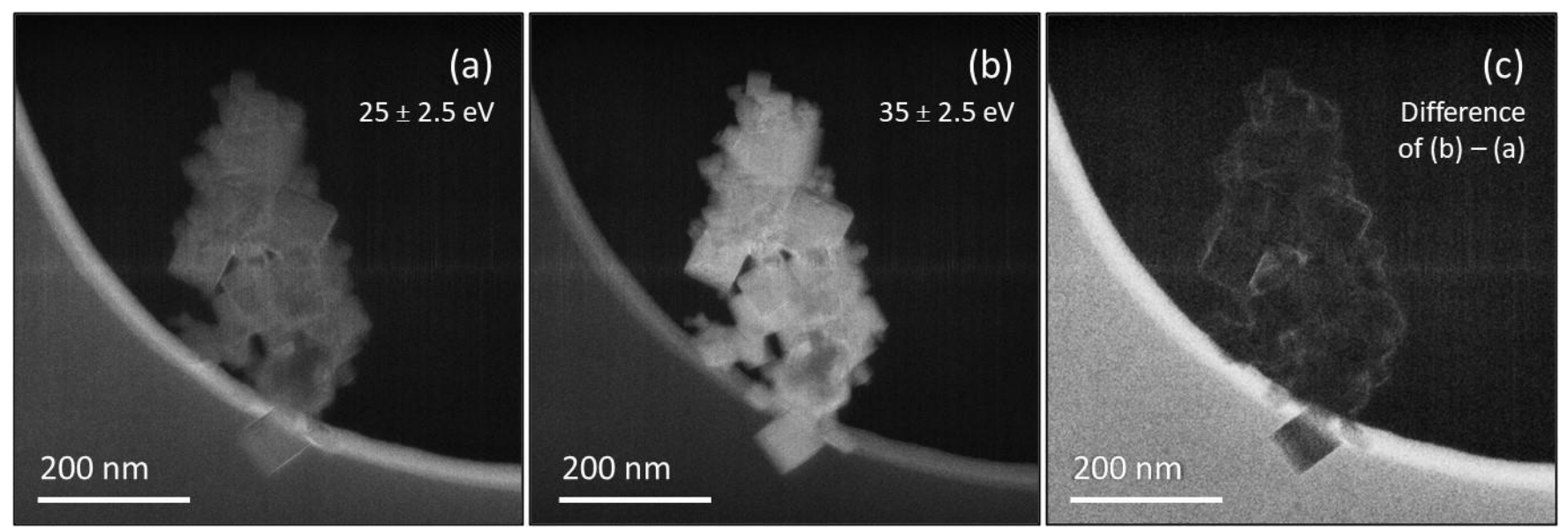

Figure 2. Figure 2. Energy-filtered images from cluster of $\mathrm{CeO} 2$ cubes recorded from area $680 \times 680 \mathrm{~nm}$ with pixel size of $0.2 \mathrm{~nm}$ (a) $25 \mathrm{eV}$ loss, b) $35 \mathrm{eV}$ loss c) surface loss after subtracting bulk contribution.

\section{References}

1. Crozier, P.A. and B.K. Miller, in Controlled Atmosphere Transmission Electron Microscopy T.W. Hansen and J.B. Wagner, Editors. 2015, Springer: New York.

2. Venkatraman, K., et al., Nature Physics, 2019. 15(12): p. 1237-1241.

3. Crozier, P.A., Ultramicroscopy, 2017. 180: p. 104-114.

4. Krivanek, O.L., et al., Nature, 2014. 514: p. 209-212.

5. $\quad$ Hage, F.S., et al., Science, 2020. 367(6482): p. 1124-+.

6. Rez, P. and A. Singh, Ultramicroscopy, 2020: p. 113162.

7. Venkatraman, K. and P.A. Crozier, Microscopy and Microanalysis (in revision), 2020.

8. Liu, Q., et al., Physical Review B, 2019. 99(16): p. 165102.

9. $\quad$ Crozier, P.A., Ultramicrosopy, 1995. 58: p. 157-174.

10 We gratefully acknowledge support of NSF grants CBET-1604971, CHE-1508667, and U.S. DOE (DE-SC0004954), and the use of (S)TEM at Eyring Materials Center at Arizona State University is gratefully acknowledged. 